\title{
Levels of plasma homocysteine in pseudoexfoliation glaucoma
}

\author{
Laura Tranchina $\cdot$ Marco Centofanti $\cdot$ Francesco Oddone $\cdot$ Lucia Tanga \\ Gloria Roberti • Laura Liberatoscioli • Claudio Cortese • Gianluca Manni
}

Received: 14 April 2010 /Revised: 26 July 2010 /Accepted: 3 August 2010 / Published online: 26 August 2010

(C) Springer-Verlag 2010

\begin{abstract}
Background To examine levels of serum homocysteine (Hcy), vitamin B12 and folic acid in patients with pseudoexfoliation glaucoma (PEXG), primary open-angle glaucoma (POAG), and healthy control subjects.

Methods This study included 36 patients with PEXG, 40 with POAG, and 40 age-matched healthy subjects. Fasting plasma Hcy concentrations and levels of serum vitamin B12 and folic acid were measured using competitive chemiluminescent enzyme immunoassay; values exceeding $14 \mu \mathrm{m} / \mathrm{l}$ were considered elevated.

Results Mean plasma Hcy was significantly higher in PEXG $(16.55 \pm 7.23 \mu \mathrm{m} / \mathrm{l})$ compared with POAG $(13.91 \pm 3.61 \mu \mathrm{m} / \mathrm{l})$ and controls $(13.12 \pm 5.13 \mu \mathrm{m} / \mathrm{l}) \quad(p=0.03$ and $p=0.0007$ respectively).

There were no statistical differences in serum vitamin B12 and folic acid levels among PEXG, POAG and control subjects $(p>0.05)$. A moderate, although statistically significant, relationship between Hcy and folic acid levels was
\end{abstract}

L. Tranchina $(\bowtie) \cdot$ M. Centofanti $\cdot$ G. Roberti $\cdot$ G. Manni Department of Biopathology, Ophthalmology Division, University of Rome "Tor Vergata" and Fondazione PTV "Policlinico Tor Vergata",

Via Montpellier,

1 - 00133 Rome, Italy

e-mail: laura3661982@libero.it

M. Centofanti $\cdot$ F. Oddone $\cdot$ L. Tanga $\cdot$ G. Manni

Fondazione G.B. Bietti-IRCCS,

Rome, Italy

L. Liberatoscioli $\cdot$ C. Cortese

Department of Internal Medicine,

University of Rome "Tor Vergata" and Fondazione PTV

"Policlinico Tor Vergata",

Rome, Italy found in the PEXG group $\left(\mathrm{R}^{2}=0.23, p=0.003\right)$. Hcy levels were found not to be related with folic acid or vitamin B12 in either POAG or control subjects.

Conclusions In this study, plasma Hcy is significantly higher in PEXG group than the POAG and control groups. Hyper-Hcy might play a role in the pathogenesis of PEXG. Hyper-Hcy may be an independent factor stressing vasculopathy in addition to pseudoexfoliation, so might be a modifiable risk factor for PEXG.

Keywords Homocysteine Pseudoexfoliation glaucoma . Hyperhomocysteinemia

\section{Introduction}

Pseudoexfoliation syndrome (PEX) is an age-related, generalized disorder of the extracellular matrix characterized by the multifocal production and progressive accumulation of a fibrillar extracellular material in intra- and extraocular tissue, which is the result of either an excessive production or insufficient breakdown or both [1].

Active involvement of the trabecular meshwork in this matrix process may lead to glaucoma development in about half of patients with PEX [2], but the exact mechanisms underlying the development of PEX and the subsequent progression to PEX glaucoma (PEXG) remain unknown [3].

Although a cause-and-effect relationship of PEXG and other systemic diseases has not been established, increasing evidence suggests that PEX is associated with cardiovascular and cerebrovascular diseases [4].

Whereas intraocular pressure is assumed to be the most important risk factor for glaucoma, there is accumulating evidence that vascular risk factors may also play a role [5, 6]; factors that lead to vascular dysregulation may contrib- 
ute to the pathogenic process [7], so impaired microcirculation and abnormal perfusion may cause optic nerve head ischemia [8].

The association between primary open-angle glaucoma (POAG) and cardiovascular disease [7] has recently stimulated interest in the role of homocysteine (Hcy) in the pathogenesis of glaucoma [9].

Hcy, a sulphur-containing amino acid synthesized during the metabolism of methionine, is a well-known independent risk factor for vascular diseases, and may contribute to ischemic alterations and oxidative stress [6]. Vitamin B12 and folic acid, which are required as co-factors in the Hcy metabolism, are non-genetic determinants of the plasma Hcy level; an alteration in either of these co-factors might lead to elevated Hcy plasma levels [10].

PEX and hyper-Hcy share common associations with various disorders.

In the Blue Mountains Eye Study, PEX is correlated positively with a history of hypertension, angina, myocardial infarction, or stroke, suggestive of vascular effects of the disease [11].

Mild hyper-Hcy is an independent risk factor for premature vascular disease, myocardial infarction, and stroke $[12,13]$, and may increase the risk of retinal vascular disease, such as retinal artery and vein occlusion [14]and nonarteritic anterior ischemic optic neuropathy [15].

The role of Hcy has been investigated in context with PEXG and POAG; most studies on PEXG have found an association with Hcy [16-19, 23, 25-27] despite some conflicting reports present in the literature [28], while only a few studies have confirmed the association in POAG patients $[9,19,20]$.

The purpose of the present study was to examine levels of serum Hcy, vitamin B12 and folic acid in patients with PEXG, POAG, and healthy control subjects.

\section{Methods}

A total of 116 subjects participated in this study, including 36 with PEXG, 40 with POAG, and 40 control subjects.

All patients were enrolled between November 2008 and March 2009, and were seen at the Department of Ophthalmology of Tor Vergata, University of Rome.

The study was conducted in accordance with the Declaration of Helsinki, and informed consent was obtained from all subjects.

Subjects were included if they had features of PEXG or POAG in one or both eyes.

PEXG was defined as the occurrence of typical exfoliation material on the anterior lens capsule in the presence of characteristic glaucomatous optic disc damage (presence of neuroretinal rim thinning, excavation, notching, or character- istic retinal nerve fiber layer defects), repeatable glaucomatous visual field defects on standard automated perimetry (Swedish Interactive Threshold Algorithm [SITA] strategy, program 24-2, Humphrey Field Analyzer), or both.

Subjects with POAG were those in the presence of glaucomatous optic disc damage and field changes as defined above with an IOP $>21 \mathrm{~mm} \mathrm{Hg}$ at the time of diagnosis without other contributing pathology.

Glaucomatous visual field loss was defined as two consecutive reliable visual fields with the glaucoma hemifield test result outside normal limits, mean deviation (MD) and pattern standard deviation (PSD) outside 95\% confidence limits, and a cluster of at least 3 points with $p<0.05$ in the pattern standard deviation plot, one of each with $p<$ 0.01 affecting the same hemifield; the cluster could not be contiguous with the blind spot, and could not cross the horizontal midline.

Control subjects had no history of elevated IOP, normal optic disc and visual fields, and no exfoliation material on the anterior lens capsule. These were spouses of patients with exfoliation or open-angle glaucoma.

Each control subject underwent a complete ophthalmological phenotypization; if a potential control had either IOP $>21 \mathrm{~mm} \mathrm{Hg}$, optic disc suspicious for glaucoma, or typical pseudoexfoliative material on the lens or anterior chamber, he or she was not recruited into the study, and was advised to seek further medical advice.

Common exclusion criteria were any history of ocular diseases (chronic or recurrent inflammatory eye disease, ocular trauma, ocular infection, severe retinal disease, corneal abnormality preventing reliable applanation tonometry), intraocular surgery within the past 12 months, or laser surgery within the past 3 months. Hcy may be influenced by many environmental factors including intercurrent disease and use of certain medications [21], so we excluded patients with medical history of disorders associated with increased Hcy levels such as thromboembolic, renal, hepatic, gastrointestinal, or neurologic diseases, and diabetes mellitus.

A detailed medical history was obtained from all enrolled subjects to determine those with known or suspected diabetes mellitus, systemic hypertension, peripheral or coronary disease, cerebrovascular disease, and other current drug therapy.

All patients underwent a complete eye examination, including best-corrected visual acuity (logMAR chart), Humphrey Field Analyser perimetry (24-2 SS), slit-lamp examination of the anterior segment, gonioscopic evaluation of the anterior chamber angle, pachymetry, IOP measurement by Goldmann applanation tonometry, and fundoscopy.

Subjects fulfilling eligibility criteria underwent a blood test for Hcy, vitamin B12, and folic acid.

Two blood samples were collected from all participants; one was placed immediately on ice for the Hcy assay. After transportation to the laboratory, both samples were 
promptly centrifuged (3000 rpm, $5 \mathrm{~min}$ ) within $1 \mathrm{hr}$ after collection, and the obtained serum was stored at $-80^{\circ} \mathrm{C}$. Biochemical analysis was performed after completion of the collection process.

Fasting plasma Hcy concentrations and levels of serum vitamin B12 and folic acid were measured using competitive chemiluminescent enzyme immunoassay (ADVIA Centaur, Switzerland) [22].

For these assays, the normal serum values of Hcy are 3.7$14 \mu \mathrm{m} / 1$, and of vitamin B12 and folic acid are, respectively, 211-911 pg/ml and 2-9 ng/ml, according to manufacturer's instructions [22].

Hyper-Hcy was defined as total plasma Hcy level $\geq 14 \mu \mathrm{m} / 1$.

\section{Statistical analysis}

Mean \pm standard deviation was used to describe continuous data.

Analysis of variance (ANOVA) was used to detect differences in continuous variables (Hcy, vitamin B12 and folic acid) among the three study groups; Student Newman-Keuls test post-hoc analysis was used. The relationship between Hcy levels and folic acid/vitaminB12 levels was explored by linear regression analysis. A $p$ value $<0.05$ was accepted as significant for all statistical tests. Statistical analyses were performed by $\mathrm{JMP}^{\circledR}$ software (Version 7., SAS Institute Inc., Cary, NC, USA).

\section{Results}

Thirty-six patients with PEXG, 40 with POAG, and 40 agematched healthy subjects were included in this study. Baseline characteristics of study subjects are shown in Table 1.

Mean \pm standard deviation (SD) of Hcy levels in PEXG was $16.55 \pm 7.23 \mu \mathrm{m} / \mathrm{l}$. Mean $\pm \mathrm{SD}$ of Hcy levels in POAG and controls were $13.91 \pm 3.61$ and $13.12 \pm 5.13 \mu \mathrm{m} / \mathrm{l}$, respectively. Hcy levels were higher in PEXG than in the controls and the POAG group $(p=0.0007$ and $p=0.03$ respectively, Fig. 1).

The mean Hcy concentrations in the POAG and control groups were not significantly different $(p=0.56)$.

Mean \pm SD of vitamin B12 levels in PEXG, POAG and control subjects were $434.55 \pm 141.46,444.90 \pm 167.17$, and $397.15 \pm 118.68 \mathrm{pg} / \mathrm{ml}$ respectively (Fig. 2). There was no significant difference in serum vitamin B12 levels between PEXG and POAG $(p=0.74)$, PEXG and controls $(p=0.25)$, or POAG and controls $(p=0.15)$.

Mean \pm SD of folic acid levels in PEXG, POAG and control subjects were $6.56 \pm 3.19,6.99 \pm 3.42$, and $7.44 \pm$ $3.86 \mathrm{ng} / \mathrm{ml}$ respectively (Fig. 3). There was no significant difference in serum folic acid levels between PEXG and POAG $(p=0.62)$, PEXG and controls $(p=0.33)$, or POAG and controls $(p=0.61)$.

A moderate, although statistically significant, relationship between Hcy and folic acid levels was found in the PEXG group $\left(\mathrm{R}^{2}=0.23, p=0.003\right)$. Hcy levels were found not to be related with folic acid or vitamin $\mathrm{B} 12$ in either POAG or control subjects $\left(\mathrm{R}^{2}<0.07, p>0.09\right)$

\section{Discussion}

In this study, plasma Hcy was significantly higher in the PEXG group than in the POAG and the control groups.

We observed that serum Hcy level in patients with POAG did not statistically differ from that of the control group. The vitamin B12/ folate status was also investigated, and showed no significant differences among the PEXG, POAG and control groups. Nevertheless, a moderate inverse relationship was detected between folic acid levels and Hcy in the PEXG group.

It is interesting that although folic acid and vitamin B12 levels were not statistically different among the three groups, PEXG and POAG presented similar folic acid and vitamin B12 levels, while control subjects were found to have somewhat lower vitamin B12 and higher folic acid levels.
Table 1 Baseline data of the study groups

PEXG $=$ Pseudoexfoliation glaucoma; $P O A G=$ Primary open angle glaucoma

\begin{tabular}{llll}
\hline & PEXG $(n=36)$ & POAG $(n=40)$ & Controls $(n=40)$ \\
\hline $\begin{array}{l}\text { Age } \\
\text { Mean } \pm \text { SD }\end{array}$ & $69.58 \pm 5.92$ & $68.71 \pm 8.65$ & $69.23 \pm 7.21$ \\
Gender & & & \\
Male, $n=(\%)$ & $25(69)$ & $22(55)$ & $21(52.5)$ \\
Hypertension & $61 ., 1 \%$ & $42.5 \%$ & $30 \%$ \\
Hyperlipidemia & $8.3 \%$ & $22.5 \%$ & $40 \%$ \\
Peripheral or coronary artery disease & $16.6 \%$ & 0 & $5 \%$ \\
Cerebrovascular disease & $2.7 \%$ & 0 & 0 \\
\hline
\end{tabular}




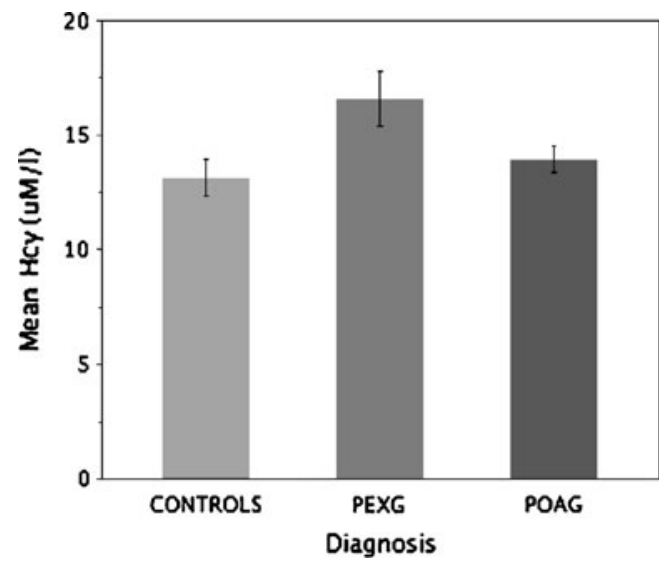

Fig. 1 Mean ( \pm standard error) of homocysteine $(H c y)$ in pseudoexfoliation glaucoma $(P E X G)$, control subjects (CONTROLS), and primary open-angle glaucoma $(P O A G)$

The effects of environmental factors, such as vitamin status, on plasma Hcy levels are complex. Whilst vitamin B12 deficiency remains the single most important environmental determinant of elevated plasma Hcy, the effects of small fluctuations in B group vitamins and folate appear minor [21]. This is highlighted by our own data, which shows significantly different mean plasma Hcy levels in PEXG patients and POAG patients, despite comparable levels of folate and vitamin B12. Similarly, POAG patients and control patients had similar mean plasma Hcy levels, despite the latter group having lower vitamin B12 and higher folate measurements. In fact, the only significant correlation identified between vitamin status and plasma Hcy was the effect of folate in the PEXG. Interestingly, Cumurcu et al. [23] similarly reported a reduced folate level in patients with PEXG and elevated Hcy compared with POAG and control. This raises the possibility that folate

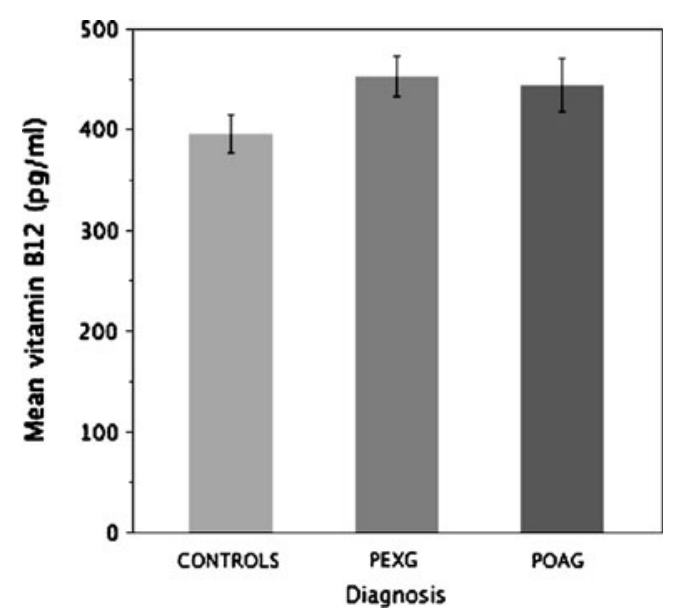

Fig. 2 Mean ( \pm standard error) of vitamin B12 in pseudoexfoliation glaucoma (PEXG), control subjects (CONTROLS), and primary openangle glaucoma $(P O A G)$

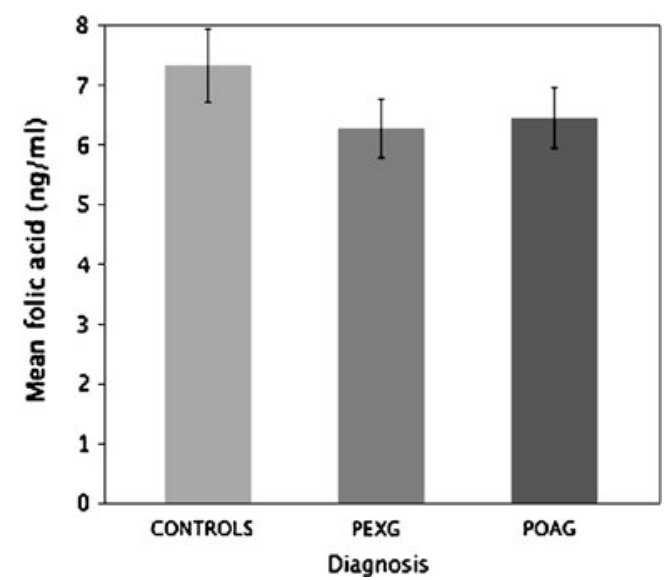

Fig. 3 Mean ( \pm standard error) of folic acid in pseudoexfoliation glaucoma $(P E X G)$, control subjects (CONTROLS), and primary openangle glaucoma (POAG)

metabolism and/or its effect on plasma Hcy is altered in PEXG patients. This warrants further investigation.

The results about plasma Hcy level in different type of glaucoma are conflicting, and differ greatly between various studies.

Different characteristics of the included populations such as age, ethnicity, severity of glaucoma, nutritional status, and presence of associated illnesses may account for the conflicting results. Also, different methodologies [24] used to assess plasma Hcy levels may explain at least part of the different results and differences in study design, such as prospective vs retrospective, inclusion and exclusion criteria, number of subjects enrolled.

An elevated plasma Hcy level may occur as the result of inherited disorders of the enzymes of its metabolism [24], but also by other conditions such as age, gender, renal failure, and medications. In the current study, an attempt was made to exclude possible confounding factors by selecting patients and controls without the above-mentioned conditions.

The mean Hcy level for subjects included in the present analysis was different than those reported in previous studies evaluating Hcy as a risk factor for glaucoma [9, 16-20, 23, 24, 26-28]; the presence of a large number of factors influencing plasma levels of Hcy makes the comparisons among different studies difficult.

The findings of this study confirm that PEXG is associated with elevated plasma Hcy levels [16-19, 23, 25-27].

A small number of studies have investigated the relationship between Hcy and POAG with different and inconsistent results.

We found that serum Hcy level in patients with POAG did not statistically differ from the control group, and this finding is in agreement with previous published works [26, 27].

In contrast, a number of studies have reported elevated plasma Hcy in patients with POAG [9, 19, 20]. In one [9], thermolabile methylenetetrahydrofolate reductase deficiency 
was thought to be causal, whereas another study [19] found no correlation. The latter study also reported elevated plasma Hcy in PEXG and normal tension glaucoma (NTG), but again found no association with methylenetetrahydrofolate reductase deficiency.

Although POAG is considered in part an ocular vascular disorder [7], the present data suggest that there is no association between elevated plasma Hcy and POAG, so other factors should be sought to elucidate vascular effects in POAG. Further, prospective studies with higher numbers of subjects with POAG will be needed to highlight the conflicting results in the literature.

On the other hand, the results of this study lead one to hypothesize that hyper-Hcy might participate in the pathogenesis of PEX.

Increasing evidence suggests that PEX is associated with cardiovascular and cerebrovascular diseases [4], while large epidemiologic studies have determined a relationship between plasma Hcy concentration and cardiovascular disease [21].

The exact mechanism of action of Hcy remains to be elucidated; it induces apoptotic cell death of retinal ganglion cells [29] and also exerts gliotoxic effects, thus representing a hypothetical risk factor for glaucoma.

Furthermore, Hcy causes dysregulation of matrix metalloproteinases and their inhibitors [30], which has been implicated in the pathogenesis of PEXG. This has led to the assumption that Hcy may also participate in the pathogenesis of PEXG.

In addition, PEX material contains elastin and fibrillin $[3,31]$, whereas Hcy activates in vitro a serine elastase, and induces elastolysis of elastin and fibrillar collagen [32].

However, the Hcy concentrations used in many of these experiments were in the pharmacologic range, and may not be relevant to the levels encountered in clinical practice.

The conflicting results of this and other studies pose considerable problems in terms of the significance of association between plasma Hcy and risk of glaucoma onset or progression, so they must, therefore, be interpreted with caution.

Hyper-Hcy may be an independent factor stressing vasculopathy in addition to PEX.

Hcy might be a modifiable factor for PEXG. Elevated plasma Hcy is readily reversible in most patients by taking vitamin preparations containing folic acid; a study demonstrated that dietary folic acid $(0.5-5 \mathrm{mg} /$ day $)$ reduces basal Hcy levels by $25 \%$ [33]. The treatment is available, inexpensive and with a low side-effect profile; this approach should motivate further research to evaluate the benefits of routine measurement of Hcy in PEXG/PEX and the influence of hyper-Hcy treatment in the development or progression of PEX.

In conclusion, hyper-Hcy might play a role in the pathogenesis of PEXG; possible mechanisms are vascular endothelial dysfunction, elastinolysis, and dysregulation of matrix metalloproteinases and their inhibitors [10, 32], but it is not clear whether elevated plasma Hcy contributes to disease development or progression.

Further large-scale studies are needed to demonstrate if Hcy measurement may be helpful to identify high-risk glaucoma populations and to have a real impact on the management of the disease.

\section{References}

1. Ritch R, Schlotzer-Schrehardt U (2001) Exfoliation syndrome: toward a new understanding: proceedings of the First International Think Tank. Acta Ophthalmol Scand 79:213-217

2. Ritch R (1994) Exfoliation syndrome: the most common identifiable cause of open-angle glaucoma. J Glaucoma 3:176-178

3. Ritch R, Schlotzer-Schrehardt U (2001) Exfoliation syndrome. Surv Ophthalmol 45:265-315

4. Schlotzer-Schrehardt U, Naumann GO (2006) Ocular and systemic pseudoexfoliation syndrome. Am J Ophthalmol 141:921-937

5. Hayreh SS (2001) Blood flow in the optic nerve head and factors that may influence it. Prog Retin Eye Res 20:595-624

6. Weinreb RN, Cioffi GA, Harris A (1997) Optic nerve blood flow. In: Van Buskirk EM, Shields B (eds) 100 Years of Progress in Glaucoma. Lippincott-Raven, Philadelphia, pp 59-78

7. Lee DJ, Gomez-Marin O, Lam BL (2003) Glaucoma and survival: the national health interview survey 1986-1994. Ophthalmology 110:1476-1483

8. Flammer J, Orgul S, Costa VP, Orzalesi N, Krieglstein GK, Serra LM, Renard JP, Stefánsson E (2002) The impact of ocular blood flow in glaucoma. Prog Retin Eye Res 21:359-393

9. Bleich S, Naumann GOH, Kornhuber J (2002) Homocysteine and risk of open angle glaucoma. J Neural Transm 109:1499-1504

10. Finklestein JD (1998) The metabolism of homocysteine: pathways and regulation. Eur J Pediatr 157:40-44

11. Mitchell P, Wang JJ, Smith W (1997) Association of pseudoexfoliation with increased vascular risk. Am J Ophthalmol 124:685687

12. Stampfer MJ, Willet WC (1992) A prospective study of plasma homocysteine and risk of myocardial infarction in US physicians. JAMA 268:877-881

13. Perry IJ, Morris RW (1995) Prospective study of serum total homocysteine concentration and risk of stroke in middle-aged British men. Lancet 346:1395-1398

14. Vine AK (2000) Hyperhomocysteinemia: a risk factor for central retinal vein occlusion. Am J Ophthalmol 129:640-644

15. Pianka P, Almog Y, Man O, Goldstein M, Sela BA, Loewenstein A (2000) Hyperhomocysteinemia in patients with nonarteritic anterior ischemic optic neuropathy, central retinal artery occlusion, and central retinal vein occlusion. Ophthalmology 107:1588-1592

16. Bleich S, Roedl J, Von Ahsen N, Schlötzer-Schrehardt U, Reulbach U, Beck G, Kruse FE, Naumann GO, Kornhuber J, Jünemann AG (2004) Elevated homocysteine in aqueous humor of patients with pseudoexfoliation glaucoma. Am J Ophthalmol 138:162-164

17. Leibovitch I, Kurtz S, Shemesh G, Goldstein M, Sela BA, Lazar M, Loewenstein A (2003) Hyperhomocysteinemia in pseudoexfoliation glaucoma. J Glaucoma 12:36-39

18. Vessani RM, Ritch R, Liebmann JM, Jofe M (2003) Plasma homocysteine is elevated in patients with exfoliation syndrome. Am J Ophthalmol 136:41-46 
19. Clement CI, Goldberg I, Healey PR, Graham SL (2009) Plasma homocysteine, MTHFR gene mutation, and open-angle glaucoma. J Glaucoma 18:73-78

20. Michael S, Qamar R, Akhtar F, Khan WA, Ahmed A (2009) MTHFR gene C677T and A1298C polymorphisms and homocysteine levels in primary open angle and primary closed angle glaucoma. Molecular Vision 15:1090

21. Refsum H, Smith AD, Ueland PM, Nexo E, Clarke R, McPartlin J, Johnston C, Engbaek F, Schneede J, McPartlin C, Scott JM (2004) Facts and recommendations about total homocysteine determinations: an expert opinion. Clin Chem 50:3-32

22. Demuth K, Ducros V, Michelsohn S, Paul JL (2004) Evaluation of Advia Centaur automated chemiluminescence immunoassay for determining total homocysteine in plasma Clin Chim Acta 349(12): $113-120$

23. Cumurcu T, Sahin S, Aydin E (2006) Serum homocysteine, vitamin B12 and folic acid levels in different types of glaucoma. BMC Ophthalmol 6:6

24. Frosst P, Blom HJ, Milos R, Goyette P, Sheppard CA, Matthews RG, Boers GJ, den Heijer M, Kluijtmans LA, van den Heuvel LP (1995) A candidate genetic risk factor for vascular disease: a common mutation in methylenetetrahydrofolate reductase. Nat Genetics 10:111-113

25. Puustjärvi T, Blomster $H$, Kontkanen $M$, Punnonen K, Teräsvirta M (2004) Plasma and aqueous humour levels of homocysteine in exfoliation syndrome. Graefes Arch Clin Exp Ophthalmol 242:749-754

26. Altintaş O, Maral H, Yüksel N, Karabaș VL, Dillioğlugil MO, Cağlar Y (2005) Homocysteine and nitric oxide levels in plasma of patients with pseudoexfoliation syndrome, pseudoexfoliation glaucoma, and primary open-angle glaucoma. Graefes Arch Clin Exp Ophthalmol 243(7):677-683

27. Turgut B, Kaya M, Arslan S, Demir T, Güler M, Kaya MK (2010) Levels of circulating homocysteine, vitamin B6, vitamin B12, and folate in different types of open-angle glaucoma. Clin Interv Aging 26(5):133-139

28. Turaçli ME, Tekeli O, Ozdemir F, Akar N (2005) Methylenetetrahydrofolate reductase $677 \mathrm{C}$ - T and homocysteine levels in Turkish patients with pseudoexfoliation. Clin Experiment Ophthalmol 33(5):505-8

29. Moore P, El-sherbeny A, Roon P, Schoenlein PV, Ganapathy V, Smith SB (2001) Apoptotic cell death in the mouse retinal ganglion cell layer is induced in vivo by the excitatory amino acid homocysteine. Exp Eye Res 73:45-57

30. Tyagi SC, Lominadze D, Roberts AM (2005) Homocysteine in microvascular endothelial cell barrier permeability. Cell Biochem Biophys 43:37-44

31. Naumann GO, Schlotzer-Schrehardt U, Kuchle M (1998) Pseudoexfoliation syndrome for the comprehensive ophthalmologist. Intraocular and systemic manifestations. Ophthalmology 105:951-968

32. Brunelli T, Prisco D, Fedi S, Rogolino A, Farsi A, Marcucci R, Giusti B, Pratesi C, Pulli R, Gensini GF, Abbate R, Pepe G (2000) High prevalence of mild hyperhomocysteinemia in patients with abdominal aortic aneurysm. J Vasc Surg 32:531-536

33. Hankey GJ, Eikelboom JW (1999) Homocysteine and vascular disease. Lancet 357:407-413 
Reproduced with permission of the copyright owner. Further reproduction prohibited without permission. 